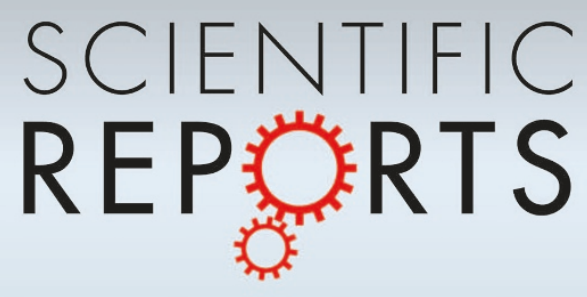

OPEN

SUBJECT AREAS:

PHYSICS

CONDENSED-MATTER PHYSICS

SUPERCONDUCTORS

SPINTRONICS

Received

11 July 2012

Accepted

5 November 2012

Published

4 December 2012

Correspondence and requests for materials should be addressed to

Z.B.H. (huangzb@ hubu.edu.cn)

\section{Magnetic instability and pair binding in aromatic hydrocarbon superconductors}

\author{
Zhongbing Huang ${ }^{1,2}$, Chao Zhang ${ }^{1}$ \& Hai-Qing Lin ${ }^{1}$
}

'Beijing Computational Science Research Center, Beijing 100084, China, ${ }^{2}$ Faculty of Physics and Electronic Technology, Hubei University, Wuhan 430062, China.

Understanding magnetism and electron correlation in many unconventional superconductors is essential to explore mechanism of superconductivity. In this work, we perform a systematic numerical study of the magnetic and pair binding properties in recently discovered polycyclic aromatic hydrocarbon $(\mathrm{PAH})$ superconductors including alkali-metal-doped picene, coronene, phenanthrene, and dibenzopentacene. The $\pi$-electrons on the carbon atoms of a single molecule are modelled by the one-orbital Hubbard model, and the energy difference $\epsilon$ between carbon atoms with and without hydrogen bonds is taking into account. We demonstrate that the spin polarized ground state is realized for charged molecules in the physical parameter regions, which provides a reasonable explanation of local spins observed in PAHs. In alkali-metal-doped dibenzopentacene, our results show that electron correlation may produce an effective attraction between electrons for the charged molecule with one or three added electrons.

ro he recent discovery of superconductivity in the solid state of various alkali-metal-doped PAHs, including picene $^{1}$, coronene $e^{2}$, phenanthrene $e^{3,4}$, and 1,2:8,9-dibenzopentacene ${ }^{5}$, has attracted a growing interest in the condensed matter physics community. One of the most intriguing properties is that the superconducting transition temperature $\mathrm{T}_{c}$ increases dramatically with increasing the number of benzene rings, reaching up to 33.1 $K$ as reported in alkali-metal-doped 1,2:8,9-dibenzopentacene ${ }^{5}$, comparable to that of alkali-metal-doped $C_{60}{ }^{6-8}$. This behavior suggests that PAHs with longer chains of benzene rings may exhibit higher $\mathrm{T}_{c}$. Another interesting experimental finding is that the magnetic susceptibility in the normal state of superconducting compounds is considerable large, and shows the Curie-Weiss behavior as a function of temperature ${ }^{1,3}$, evidencing that there exist local spins in the charge doped systems. Currently, the physical origin of local spins is unclear.

Theoretical studies mainly based on the first-principle calculations have been carried out to elucidate the superconducting mechanism in these PAH superconductors. There are three important findings: (1)The conduction band group around the Fermi level comprises four bands, which are derived from the lowest unoccupied molecular orbital (LUMO) and LUMO+ $1^{9-11}$. (2) The bandwidth $W$ around the Fermi energy is smaller than the effective Coulomb interaction $U_{\text {eff }}$ in alkali-doped picene, placing it in the strong correlation regime ${ }^{12,13}$. (3) Intramolecular ${ }^{14,15}$, intermolecular, and intercalant ${ }^{16}$ phonons are shown to have significant contribution to the electron-phonon interaction in alkali-doped picene. However, Kato et al. ${ }^{17}$ found that the electron-phonon coupling constant decreases with an increase of the number of carbon atoms in phenanthrene edge-type hydrocarbons, leading to a decrease of $\mathrm{T}_{c}$ with increasing size of phenanthrene edge-type hydrocarbons. This contradicts to the experimental results that $\mathrm{T}_{c}$ increases from $\sim 5 K$ in phenanthrene to $\sim 33 K$ in $1,2: 8,9$ dibenzopentacene. Finding (3) indicates that the superconductivity in PAHs can not be explained by considering only the electron-phonon interaction. Finding (2) suggests that electronic correlations are required to be taken into account for a complete understanding of the physical properties of PAHs.

To gain some insights on the effect of electronic correlations on the magnetic and pair binding properties of PAHs, we undertake a first step to study the electronic states on a single molecule, which provides an energy scale intermediate between the microscopic energetics at the level of carbon atom and the macroscopic scale of the molecular solids. The resulting many-body states form a basis for analyzing the low-energy physics at the length scale larger than the size of molecule ${ }^{18}$. The carbon atoms in PAHs are in a trigonal planar $s p^{2}$ hybridization configuration with a half-filled $p_{\pi}$ orbital perpendicular to the planar structure, which forms energy levels (including LUMO and LUMO +1 ) around the Fermi energy. The minimal description of interacting $p_{\pi}$-electrons on a single molecule is given by the one-orbital Hubbard model which incorporates an on-site Coulomb repulsion besides a simple Huckel picture for the one-electron part. The Hamiltonian reads, 


$$
H=-t \sum_{\langle i j\rangle, \sigma}\left(c_{i \sigma}^{\dagger} c_{j \sigma}+c_{j \sigma}^{\dagger} c_{i \sigma}\right)-\epsilon \sum_{i}{ }^{\prime} n_{i}+U \sum_{i} n_{i \uparrow} n_{i \downarrow},
$$

The operators $c_{i \sigma}^{\dagger}$ and $c_{i \sigma}$ create and destroy a $\pi$-electron with spin $\sigma$ at the $i$ th carbon atom of a single molecule, respectively. $n_{i \sigma}=c_{i \sigma}^{\dagger} c_{i \sigma}$ is the number operator for an electron with spin $\sigma$ at site $i$, and $n_{i}=$ $n_{i \uparrow}+n_{i \downarrow}$. The sum $\langle i j\rangle$ is over nearest-neighbor (NN) carbon atoms, and the primed sum runs over the carbon sites without connecting to hydrogen atoms. Here, $U$ denotes the on-site Coulomb interaction and $\epsilon$ the energy difference between carbon atoms with and without hydrogen bonds, which is due to different electron-negativity of carbon and hydrogen. Notice that the on-site Hubbard $U$ is different from the effective Coulomb interaction $U_{\text {eff }}{ }^{12,13}$, which stands for the energetic cost to doubly occupy a molecular orbital. Based on the estimated parameters ${ }^{18-20}: t \sim 2.5 \mathrm{eV}$ to $3.0 \mathrm{eV}$ and $U \sim 6 \mathrm{eV}$ to $10 \mathrm{eV}$, we mainly used $U=2 t$ and $3 t$ in our studies. Two accurate numerical methods, exact diagonalization (ED) ${ }^{21}$ and constrainedpath Monte Carlo (CPMC) ${ }^{22,23}$, are employed to simulate the model Hamiltonian (1). In the followings, the results presented are obtained by the CPMC method, except where explicitly noted otherwise.

\section{Results}

Four hydrocarbon molecules investigated in our studies are displayed in Fig. 1, which are defined as $\mathrm{C}_{14} \mathrm{H}_{10}$ (a), $\mathrm{C}_{30} \mathrm{H}_{18}$ (b), $\mathrm{C}_{22} \mathrm{H}_{14}-\mathrm{A}(\mathrm{c})$, and $\mathrm{C}_{22} \mathrm{H}_{14}-\mathrm{B}(\mathrm{d})$. The former three correspond to the hydrocarbon molecules in phenanthrene, $1,2: 8,9$-dibenzopentacene, and picene, respectively. $\mathrm{C}_{22} \mathrm{H}_{14}-\mathrm{B}$, which has a similar structure to $\mathrm{C}_{30} \mathrm{H}_{18}$ with both linear and angular fusion of benzene rings, is degenerate with $\mathrm{C}_{22} \mathrm{H}_{14}-\mathrm{A}$ from our first-principle calculations, suggesting its possible existence in alkali-metal-doped picene.

In the noninteracting limit, i.e. $U=0$, the electronic spectrum can be easily obtained by diagonalizing the quadratic Hamiltonian in Eq. (1). The energy difference between LUMO and LUMO+1, $\mathrm{E}(\mathrm{LUMO}+1)-\mathrm{E}(\mathrm{LUMO})$, is shown in Fig. 2 as a function of $\epsilon$ for different molecules. It is obviously that the electronic spectrum depends sensitively on the molecule structure. For $\mathrm{C}_{14} \mathrm{H}_{10}$ and $\mathrm{C}_{22} \mathrm{H}_{14}-\mathrm{A}$ with phenanthrene edge-type termination, the energy difference decreases monotonically with increasing $\epsilon$; while for $\mathrm{C}_{30} \mathrm{H}_{18}$ and $\mathrm{C}_{22} \mathrm{H}_{14}-\mathrm{B}$, it decreases first with increasing $\epsilon$, and then turns to increase with $\epsilon$ beyond a certain $\epsilon$. The energy differences between LUMO and LUMO +1 in phenanthrene and picene from the first-principle calculations are estimated to be about $0.1 \mathrm{eV}$ and $0.07 \mathrm{eV}$ (see Supplementary Information), respectively, which are consistent with the results for $\mathrm{C}_{14} \mathrm{H}_{10}$ at $\epsilon \sim 0.8 t$ and for $\mathrm{C}_{22} \mathrm{H}_{14}-$ $\mathrm{A}$ at $\epsilon \sim 0.87 t$. This indicates that PAHs could be well described by the model Hamiltonian (1) with $\epsilon>0.8 t$.

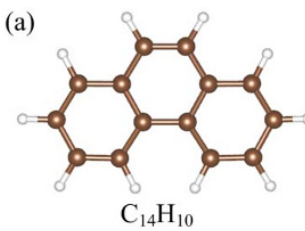

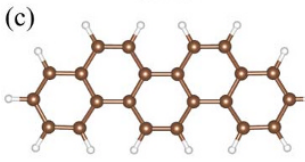

$\mathrm{C}_{22} \mathrm{H}_{14}-\mathrm{A}$ (b)

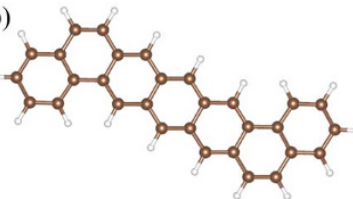

(d)

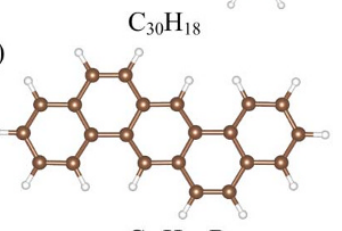

Figure 1 Sketch of aromatic hydrocarbon molecules with (a) 14 carbon atoms, (b) 30 carbon atoms, and (c) and (d) 22 carbon atoms.

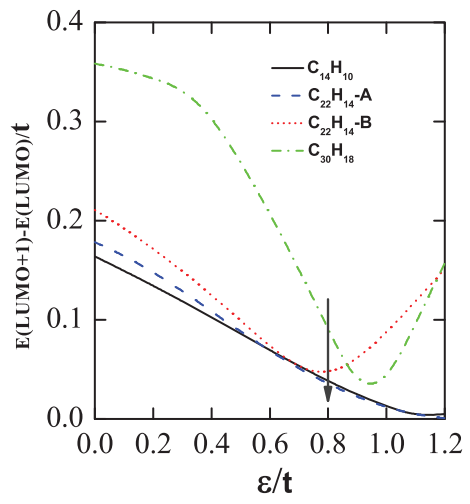

Figure $2 \mid$ Energy difference between LUMO and LUMO +1 as a function of $\epsilon$ for $\mathrm{C}_{14} \mathrm{H}_{10}$ (solid line), $\mathrm{C}_{22} \mathrm{H}_{14}-\mathrm{A}$ (dashed line), $\mathrm{C}_{22} \mathrm{H}_{14}-\mathrm{B}$ (dotted line), and $\mathrm{C}_{30} \mathrm{H}_{18}$ (dashed-dotted line). The gray arrow indicates the position where $\mathrm{E}(\mathrm{LUMO}+1)-\mathrm{E}(\mathrm{LUMO})$ is approximately $0.1 \mathrm{eV}$ for $\mathrm{C}_{14} \mathrm{H}_{10}$ and $\mathrm{C}_{22} \mathrm{H}_{14}-\mathrm{A}$.

Magnetic property. First, we show the spin phase diagram of different molecules in Fig. 3 at $U=2 t$. Here, we present the results for the neutral molecule (the total number of $p_{\pi}$-electrons $N_{e}$ equals the total number of carbon atoms $N_{C}$ ) and charged molecules with 1 , 2,3 , and 4 added electrons. The total spin $S$ of the ground state is obtained by comparing the energies in different spin sectors. The molecules not shown in Fig. 3 always lie in the lowest spin states $(S=$ 0 for even $N_{e}$ and $S=1 / 2$ for odd $N_{e}$ ). One can clearly see that for the two electrons added cases, the total spin $S$ switches from 0 to 1 at a certain critical $\epsilon$ for all molecules shown in Fig. 1. Moreover, for the molecule $\mathrm{C}_{30} \mathrm{H}_{18}$, the four electrons added case shows a transition from the high $\operatorname{spin}(S=1)$ state to the low spin $(S=0)$ state as $\epsilon$ is increased. At $U=3 t$, the phase diagram is similar to the one presented in Fig. 3. Besides, we find that $\mathrm{C}_{30} \mathrm{H}_{18}$ with three electrons added changes from being in the low spin $(S=1 / 2)$ state to the high $\operatorname{spin}(S=3 / 2)$ state at $\epsilon=0.97 t$.

The spin polarized state for the charged molecules with two added electrons at large $\epsilon$ provides a unified explanation of local spins observed for PAHs superconductors in the normal states, with an average number of added electrons per molecule $n^{\text {ave }} \sim 3^{1-5}$. In alkalimetal-doped PAHs, the added electrons come from alkali-metal atoms intercalated between the stacked molecules ${ }^{9-11}$. Therefore, local inhomogeneous intercalation of alkali-metal atoms in the molecular solid may induce a local nonuniform charge

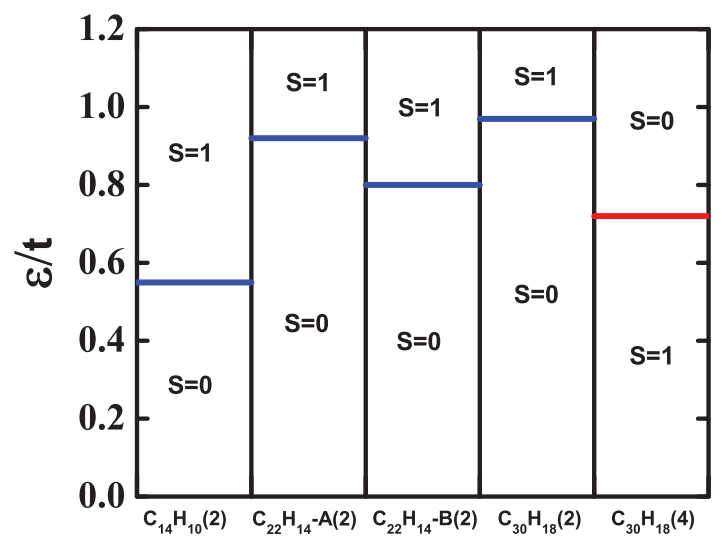

Figure 3 Spin phase diagram for charged hydrocarbon molecules as displayed in Fig. 1 at $U=2 t$. The total spin $S$ of the ground state is shown for a combination of molecule structure, charge, and $\epsilon$. The integer in the bracket denotes the number of electrons added to the neutral molecule. 
Table I $\mid V$ dependence of the energy difference $\Delta E=E(S=1)-$ $E(S=0)$ for the charged molecules with two added electrons at $U$ $=2 t$ and $\epsilon=1.0 t$. Statistical errors are in the last digit and shown in the parentheses

\begin{tabular}{lccc}
$\mathrm{V} / \mathrm{t}$ & $\Delta \mathrm{E}\left(\mathrm{C}_{14} \mathrm{H}_{10}\right)$ & $\Delta \mathrm{E}\left(\mathrm{C}_{22} \mathrm{H}_{14}-\mathrm{A}\right)$ & $\Delta \mathrm{E}\left(\mathrm{C}_{22} \mathrm{H}_{14}-\mathrm{B}\right)$ \\
\hline 0.0 & $-0.0452(6)$ & $-0.0074(8)$ & $-0.1073(7)$ \\
0.1 & $-0.0305(6)$ & $0.0009(8)$ & $-0.1041(9)$ \\
0.2 & $-0.0016(8)$ & $0.0121(9)$ & $-0.1017(9)$ \\
0.3 & $-0.0044(8)$ & $0.0257(9)$ & $-0.0965(9)$ \\
0.4 & $0.0134(9)$ & $0.043(1)$ & $-0.090(1)$ \\
0.5 & $0.0289(9)$ & $0.060(1)$ & $-0.086(1)$ \\
\hline
\end{tabular}

distribution on different molecules. If two electrons happen to lie on the same molecule, the spin polarized state is favored, which contributes to the high magnetic susceptibility observed in experiments.

Now the question arising is why the Curie-Weiss behavior of magnetic susceptibility is only observed when $n^{\text {ave }}$ is larger than $\mathrm{two}^{1,3}$. This issue can be addressed by studying the effect of long range Coulomb interaction on the ground state of charged molecules with two added electrons. An inclusion of NN Coulomb interaction $V \sum_{\langle i j\rangle} n_{i} n_{j}(V$ denotes the strength of screened NN Coulomb repulsion) to the Hamiltonian (1) indicates that the energy difference between the high spin $(S=1)$ state and the low spin $(S=0)$ state increases with increasing $V$, as shown in Table I. One can observe that this energy difference becomes positive for $V \geq 0.4 t$ and $V \geq 0.1 t$ on $\mathrm{C}_{14} \mathrm{H}_{10}$ and $\mathrm{C}_{22} \mathrm{H}_{14}-\mathrm{A}$, respectively, suggesting that the low spin state is more stable when the NN Coulomb interaction is not all screened out. Considering the fact that electronic screening decreases with decreasing the metallicity of the molecular solid, it was expected that when $n^{\text {ave }}$ is not large enough, NN Coulomb interaction is not screened out, making the low spin state more stable than the spin polarized state.

Pair binding property. In a strongly correlated electronic system, electron pairing may be induced either by a purely electronic mechanism or by the interplay of electron-electron interaction and electron-phonon coupling. The effect of electronic correlations on the superconductivity can be explored by examining the pair binding energy, which is defined as: $\Delta_{i}=2 E_{i}-E_{i-1}-E_{i+1}$ with $E_{i}$ representing the ground state energy for the charged molecule having $\mathrm{i}$ added electrons ${ }^{18}$. In this notation, a positive $\Delta_{i}$ implies that an effective attraction between added electrons can be produced through a charge disproportionation process between the two molecules: $(i)+$ $(i) \rightarrow(i-1)+(i+1)$, meaning that it is energetically favorable to have two molecules with $i-1$ and $i+1$ added electrons each rather than two molecules with $i$ added electrons each. We have calculated the pair binding energy $\Delta_{i}(i=1$ and 3$)$ for all molecules as shown in Fig. 1 and the results show that it is always negative in the whole parameter regime for $\mathrm{C}_{14} \mathrm{H}_{10}, \mathrm{C}_{22} \mathrm{H}_{14}-\mathrm{A}$ and $\mathrm{C}_{22} \mathrm{H}_{14}-\mathrm{B}$ (see Supplementary Information). In Fig. 4, we show $\Delta_{1}$ and $\Delta_{3}$ for $\mathrm{C}_{30} \mathrm{H}_{18}$ as a function of $\epsilon$. It is interesting to see that both $\Delta_{1}$ and $\Delta_{3}$ take positive values at $U=2 t$ and $U=3 t$ for $\epsilon \geq 1.0 t$, demonstrating that electron pair is favored in the $\mathrm{C}_{30} \mathrm{H}_{18}$ molecular solid at $n^{\text {ave }}=1.0$ and $n^{\text {ave }}=3.0$. The positive $\Delta_{1}$ and $\Delta_{3}$ actually represent the energy gain due to the formation of bounded $S=1$ doublycharged $\mathrm{C}_{30} \mathrm{H}_{18}$ and bounded $S=0$ quadruply-charged $\mathrm{C}_{30} \mathrm{H}_{18}$, respectively, when two electrons are added to the neutral or already doubly-charged molecules. A similar pairing process was suggested to be responsible for superconductivity seen in alkalimeta-doped $C_{60}$, but it occurs for $U>3.3 t^{18}$.

The effective attraction between added electrons resulted from the positive pair binding energy may lead to various charge densitywave, magnetic, and superconducting states, depending on the details of interactions between electrons around the Fermi level
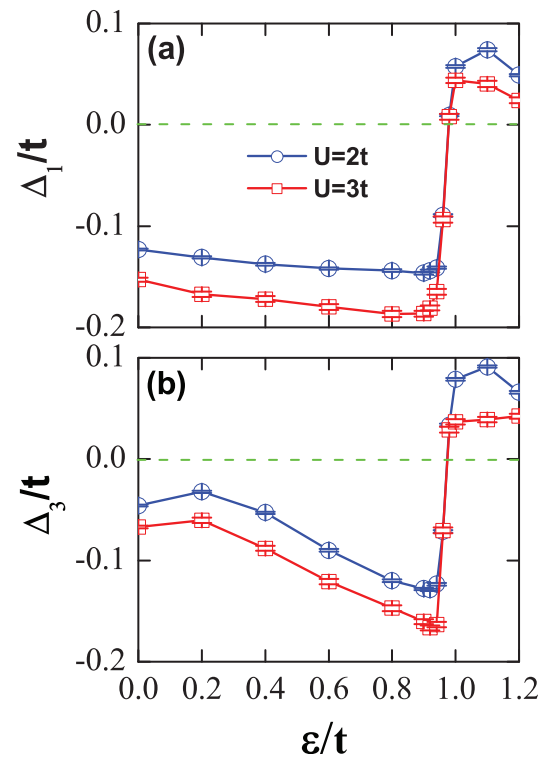

Figure $4 \mid$ Pair binding energies $\Delta_{1}\left(\right.$ a) and $\Delta_{3}\left(\right.$ b) versus $\epsilon$ for $C_{30} H_{18}$. The on-site interaction $U$ is indicated by the shape of symbol.

(including the intermolecular interaction which has not been considered in our present study $)^{24}$. In the case that instability to superconductivity is predominant, it is expected that the positive pair binding energy has a contribution to the formation of Cooper pairs. In the potassium-doped 1,2:8,9-dibenzopentacene, superconductivity was observed for $3.0 \leq n^{\text {ave }} \leq 3.5^{5}$. Therefore, a positive $\Delta_{3}$ at large epsilon suggests that electron correlation might have a contribution to the formation of Cooper pairs. The bounded $S=0$ state for quadruply-charged $\mathrm{C}_{30} \mathrm{H}_{18}$ is consistent with possible s-wave superconductivity in dibenzopentacene, of which the magnetic susceptibility shows a similar temperature dependence to the one in barium-doped phenanthrene, where s-wave superconductivity has been demonstrated by specific-heat measurements ${ }^{25}$. At $\epsilon=1$.0t, our calculations with an inclusion of nearest-neighbor Coulomb interaction $V \sum_{\langle i j\rangle} n_{i} n_{j}$ show that both $\Delta_{1}$ and $\Delta_{3}$ are increased by a finite $V$, indicating that the effective attractive interaction between electrons is robust against the long range Coulomb interaction.

\section{Discussion}

Our numerical calculations show that there exists strong magnetic instability in the charged molecules of PAHs, especially for the even number of added electrons, which provides a reasonable explanation of local spins observed in experiments. In alkali-metal-doped dibenzopentacene, electron correlation may produce an effective attraction between added electrons through charge disproportionation in different $\mathrm{C}_{30} \mathrm{H}_{18}$ molecules. This is not only useful for understanding the superconducting mechanism in alkali-metal-doped dibenzopentacene, but also encouraging for the search of higher $\mathrm{T}_{c} \mathrm{PAH}$ superconductors with longer chains of benzene rings. Experimental test on the spin state and charge distribution in PAHs can be compared with our predictions and also is crucial for clarifying the magnetic and superconducting properties of charged PAHs.

\section{Methods}

To study the magnetic and pair binding properties of PAHs, we have mainly used the CPMC method ${ }^{22,23}$ to compute the ground state of interacting $p_{\pi}$-electrons described in the model Hamiltonian (1). The basic strategy of the CPMC method is to project the ground-state wave function from an initial wave function (with correct quantum numbers corresponding to the ground state) by a branching random walk in an overcomplete space of constrained Slater determinants, which have positive overlaps with a known trial wave function. For more technique details we refer to Refs. [22, 23]. Since the total spin $S$ of the ground state is unknown a priori, we first applied the CPMC method to project the lowest eigenstates of Hamiltonian (1) in different spin 
sectors $\left(S, S^{z}\right)$, and then defined the one with lowest energy as the ground state. In our calculations, the free-electron wave function, given by Eq. (1) at $U=0$ and represented by a single Slater determinant, was chosen as the initial and trial wave functions. This choice achieves the projection in a certain spin sector with $S=\left|S^{z}\right|=\frac{1}{2}\left|N_{\uparrow}-N_{\downarrow}\right|$. Here, $N_{\uparrow}$ and $N_{\downarrow}$ denote the total numbers of electrons with spin up and spin down, respectively.

To justify the accuracy of the CPMC program, in Fig. S1 and Fig. S2 (see Supplementary Information) we compare the pair binding energy obtained from both ED and CPMC methods on $\mathrm{C}_{14} \mathrm{H}_{10}$ at $U=2 t$ and $U=3 t$. We observe that the CPMC data are in good agreement with the ED results and the systematic error induced by the constraint of Slater determinant is small. In Ref. [22], extensive benchmark calculations for the one-band Hubbard model defined on a square lattice also showed that in the weak and intermediate coupling regimes, the CPMC method can present us accurate energy and reliable correlations of the ground state.

1. Mitsuhashi, R. et al. Superconductivity in alkali-metal-doped picene. Nature 464, 76-79 (2010).

2. Kubozono, Y. et al. Metal-intercalated aromatic hydrocarbons: a new class of carbon-based superconductors. Phys. Chem. Chem. Phys. 13, 16476-16493 (2011).

3. Wang, X. F. et al. Superconductivity at $5 \mathrm{~K}$ in alkali-metal-doped phenanthrene. Nature Communications 2, 507-513 (2011)

4. Wang, X. F. et al. Superconductivity in $\mathrm{A}_{1.5}$ phenanthrene $(\mathrm{A}=\mathrm{Sr}, \mathrm{Ba})$. Phys. Rev. $B$ 84, 214523 (2011)

5. Xue, M. et al. Superconductivity above $30 \mathrm{~K}$ in alkali-metal doped hydrocarbon. Scientific Reports 2, 389-392 (2012).

6. Hebard, A. F. et al. Superconductivity at $18 \mathrm{~K}$ in potassium-doped C60. Nature 350, 600-601 (1991).

7. Ganin, A. Y. et al. Bulk superconductivity at $38 \mathrm{~K}$ in a molecular system. Nature Mater. 7, 367-371 (2008).

8. Ganin, A. Y. et al. Polymorphism control of superconductivity and magnetism in $\mathrm{Cs}_{3} \mathrm{C}_{60}$ close to the Mott transition. Nature 466, 221-225 (2010).

9. Nomura, $\mathrm{Y}$. et al. $\mathrm{Ab}$ initio derivation of electronic low-energy models for $\mathrm{C}_{60}$ and aromatic compounds. Phys. Rev. B 85, 155452 (2012).

10. Kosugi, T. et al. First-principles structural optimization and electronic structure of the superconductor picene for various potassium doping levels. Phys. Rev. B 84, 214506 (2011).

11. de Andres, P. L. et al. Ab initio electronic and geometrical structures of tripotassium-intercalated phenanthrene. Phys. Rev. B 84, 144501 (2011).

12. Giovannetti, G. \& Capone, M. Electronic correlation effects in superconducting picene from ab initio calculations. Phys. Rev. B 83, 134508 (2011).

13. Kim, M. et al. Density functional calculations of electronic structure and magnetic properties of the hydrocarbon $\mathrm{K}_{3}$ picene superconductor near the metal-insulator transition. Phys. Rev. B 83, 214510 (2011).

14. Kato, T. et al. Strong Intramolecular Electron-Phonon Coupling in the Negatively Charged Aromatic Superconductor Picene. Phys. Rev. Lett. 107, 077001 (2011).
15. Subedi, A. \& Boeri, L. Vibrational spectrum and electron-phonon coupling of doped solid picene from first principles. Phys. Rev. B 84, 020508(R) (2011).

16. Casula, M. et al. Intercalant and Intermolecular Phonon Assisted Superconductivity in K-Doped Picene. Phys. Rev. Lett. 107, 137006 (2011).

17. Kato, T. et al. Electron-phonon coupling in negatively charged acene- and phenanthrene-edge-type hydrocarbon crystals. J. Chem. Phys. 116, 3420-3429 (2002).

18. Chakravarty, S. et al. Electron Correlation Effects and Superconductivity in Doped Fullerenes. Science 254, 970-974 (1991).

19. Castro Neto, A. H. et al. The electronic properties of graphene. Rev. Mod. Phys. 81, 109-162 (2009)

20. Wehling, T. O. et al. Strength of Effective Coulomb Interactions in Graphene and Graphite. Phys. Rev. Lett. 106, 236805 (2011).

21. Lin, H. Q. \& Gubernatis, J. E. Exact Diagonalization Methods for Quantum Systems. Computers in Physics 7 (4), 400 (1993).

22. Zhang, S. W. et al. Constrained path Monte Carlo method for fermion ground states. Phys. Rev. B 55, 7464 (1997).

23. Huang, Z. B. et al. Quantum Monte Carlo study of Spin, Charge, and Pairing correlations in the $t-t^{\prime}-U$ Hubbard model. Phys. Rev. B 64, 205101 (2001)

24. Chakravarty, S. \& Kivelson, S. A. Electronic mechanism of superconductivity in the cuprates, $\mathrm{C}_{60}$, and polyacenes. Phys. Rev. B 64, 064511 (2001).

25. Ying, J. J. et al. s-wave superconductivity in barium-doped phenanthrene as revealed by specific-heat measurements. Phys. Rev. B 85, 180511(R) (2012).

\section{Acknowledgements}

This work was supported by MOST 2011CB922200, the Natural Science Foundation of China under Grants Nos. 10974047 and 11174072, and by SRFDP under Grant No. 20104208110001.

\section{Author contributions}

Z.B.H. developed the numerical codes and performed the calculations. C.Z. carried out the first-principle calculations. Z.B.H. and H.Q.L. analyzed the data, wrote the paper and led the project.

\section{Additional information}

Supplementary information accompanies this paper at http://www.nature.com/ scientificreports

Competing financial interests: The authors declare no competing financial interests.

License: This work is licensed under a Creative Commons

Attribution-NonCommercial-ShareAlike 3.0 Unported License. To view a copy of this license, visit http://creativecommons.org/licenses/by-nc-sa/3.0/

How to cite this article: Huang, Z.B., Zhang, C. \& Lin, H.Q. Magnetic instability and pair binding in aromatic hydrocarbon superconductors. Sci. Rep. 2, 922; DOI:10.1038/ srep00922 (2012) 